\title{
The role of soluble fibrin during anticoagulant therapy: a case report
}

\author{
Tohru Kawakami ${ }^{*}$, Nobukiyo Tanaka², Hiroki Ishihara² and Hiroyoshi Ohno²
}

\begin{abstract}
Warfarin, dabigatran, and apixaban are used for preventing ischemic stroke due to non-valvular atrial fibrillation (NVAF). However, it is often challenging to select the appropriate anticoagulant. We present the case of a 70-year-old male patient with persistent NVAF who developed pulmonary thromboembolism (PTE), deep vein thrombosis (DVT), and left atrial thrombus during anticoagulant therapy with warfarin. Intravenous recombinant tissue plasminogen activator was administered during his acute PTE. Heparin and apixaban were administered over 28 days; heparin was discontinued after the DVT resolved, while apixaban was administered to prevent ischemic stroke. Two days after heparin was discontinued, the patient experienced an ischemic stroke. Dabigatran was administered for secondary ischemic stroke prevention. Soluble fibrin (SF) levels remained elevated during treatment with heparin and apixaban and returned to normal after apixaban was replaced with dabigatran. Monitoring of SF may be useful as an index for selection of anticoagulants.
\end{abstract}

Keywords: Soluble fibrin, Pulmonary thromboembolism, Atrial fibrillation, Deep vein thrombosis, Warfarin, Apixaban, Dabigatran

\section{Background}

Left atrial appendage (LAA) thrombus is commonly associated with non-valvular atrial fibrillation (NVAF) and causes thromboembolic complications. Warfarin is used not only for prevention of thromboembolic complications in NVAF patients, but also for treatment of deep vein thrombosis (DVT) [1, 2]. The optimal therapeutic range for anticoagulation with warfarin to prevent thromboembolic events in NVAF patients extends over INR values of 2.0 to 3.0 [3, 4]. Recently, non-vitamin K antagonist oral anticoagulants (NOACs) have been used for preventing ischemic stroke due to NVAF. These drugs are superior to warfarin in preventing stroke or systemic embolism and cause less bleeding [5, 6]. An index of the effect of NOACs has not been established, and the challenge to physicians is how to select an appropriate anticoagulant from this group of drugs.

Recent studies have shown that soluble fibrin (SF) and D-dimer levels have been considered useful for the diagnosis of thrombosis. Soluble fibrin is generated from

\footnotetext{
* Correspondence: tkawakami-ind@umin.ac.jp

'Division of Laboratory Medicine, Ichinomiyanishi Hospital, 1 Kaimei-hira, Ichinomiya, Aichi 494-0001, Japan

Full list of author information is available at the end of the article
}

thrombin-fibrinogen reactions and is a potentially useful marker of hypercoagulability, while D-dimer is a small protein fragment and is detected only after a blood clot is degraded by fibrinolysis [7-12].

\section{Case presentation}

A 70-year-old male underwent warfarin treatment to prevent the thromboembolic complications of NVAF. He consulted our hospital due to severe dyspnea and hypotension. His warfarin treatment time in therapeutic range (TTR) was $100 \%$ during the 3 months prior to hospitalization in our institution.

His pulmonary thromboembolism (PTE) and DVT were revealed by enhanced computed tomography (CT). He underwent thrombolysis with 80,000 IU of tissue plasminogen activator (t-PA). At hospitalization, he had elevated levels of SF $(32.5 \mu \mathrm{g} / \mathrm{mL})$ and D-dimer $(35.8 \mu \mathrm{g} /$ $\mathrm{mL}$ ), with an international normalized ratio (INR) within his target therapy level (2.28), normal levels of anti cardiolipin beta2-glycoprotein I complex antibody $(<0.7 \mathrm{U} / \mathrm{mL})$ and protein C activity (98\%), and decreased protein S activity (46\%). His CT findings suggested lung cancer and LAA thrombus. After thrombolysis with t-PA, we recognized that warfarin therapy was not available for this 
patient, and we started apixaban therapy ( $5 \mathrm{mg}$ twice daily) combined with heparin (15,000 IU). We performed transesophageal echocardiography, which revealed thrombus formation in the LAA (Fig. 1a). The elevated SF and Ddimer levels decreased, but did not return to normal during treatment with apixaban and heparin.

At Day 29 of hospitalization, resolution of his DVT was confirmed with echo-venography. We discontinued heparin dosing, but continued apixaban treatment. At Day 31, the patient noted an abnormal feeling in his right hand, this symptom continued over time. At Day 33, multiple ischemic strokes were revealed using magnetic resonance imaging (Fig. 1b). Apixaban therapy was discontinued and edaravon $60 \mathrm{mg}$ was started. By Day 36 , the patient's SF and D-dimer levels were significantly elevated. We started dabigatran therapy $(110 \mathrm{mg}$ twice daily) with heparin (15,000 IU) for secondary prevention of embolism. At Day 40, his SF level had normalized $(5.6 \mu \mathrm{g} / \mathrm{mL})$ and his D-dimer level decreased $(5.5 \mu \mathrm{g} / \mathrm{mL})$ (Fig. 1c). The patient did not have a recurrence of thrombosis after dabigatran therapy with heparin, and chemotherapy (CBDCA + PEM) was started to treat his lung cancer (adenocarcinoma, T4NXM1a, stage IV) at Day 65. However, the patient expired after 79 days of hospitalization.

Note: All blood samples were collected before breakfast. During this trough period, INR, prothrombin time, $\mathrm{SF}$, and D-dimer were all detectable. All assays were performed in our institute's laboratory. For quantitative determination of coagulation tests, an automated coagulation analyzer was used (Coapresta 2000; Sekisui Medical Co., Ltd., Tokyo, Japan). Prothrombin time was measured with Coagpia PT-N (Sekisui Medical Co., Ltd.), whose international sensitivity index is almost equal to 1.0. Sodium citrate-preserved plasma was used to measure SF (Nanopia SF; Sekisui Medical Co., Ltd.) and D-dimer (Nanopia D-dimer; Sekisui Medical Co., Ltd.). Processing and analysis of all samples was performed within 90 min of sample collection. This case report was approved by the ethics committee of ichinomiyanishi hosiptal.

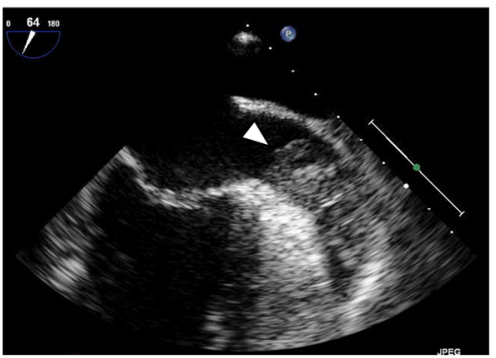

A

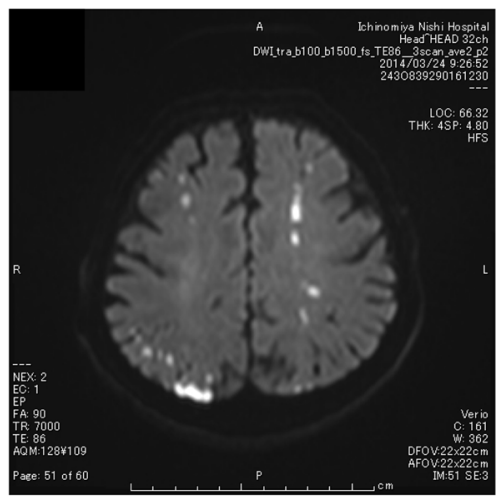

B

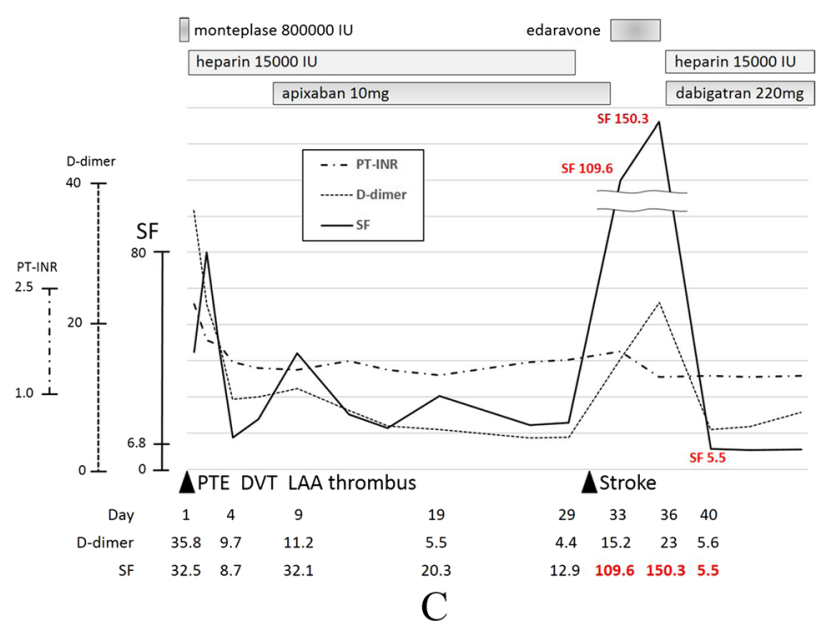

Fig. 1 Transesophageal echocardiography, MRI, laboratory data for the coagulation system and administration of medicine. a Thrombus formation (arrow) in the apex of the left atrial appendage (LAA) on Day 8. b Acute multiple ischemic stroke due to NVAF in MRI (T2-weighted image). c Plot of laboratory coagulation data demonstrates high levels of SF during apixaban and heparin therapy, and a decrease in SF starting after dabigatran and heparin therapy 


\section{Discussion}

The purpose of anticoagulant therapy is prevention of thrombosis formation. In this case, warfarin therapy with suitable TTR did not prevent thrombosis. The optimal therapeutic range for anticoagulation with warfarin in the prevention of thromboembolic events with NVAF patients extends over INR values of 2.0 to 3.0. It has been reported that major bleeding is observed only at INR values above 2.5 on anticoagulation with warfarin [3]. Warfarin therapy is evaluated by monitoring of INR level; the measurement of INR value during warfarin therapy is a suitable tool for assessment of bleeding risk, but it does not alert the physician to hypercoagulability. Apixaban and dabigatran are superior to warfarin in preventing stroke or systemic embolism, and cause less bleeding $[5,6]$.

Soluble fibrin is generated from thrombin-fibrinogen reactions, and high SF levels indicate hypercoagulability [13-15]. In a previous report, SF and D-dimer levels decreased along with LAA thrombus resolution without prolongation of INR when the patient was under apixaban therapy [16]. In this case, it was observed that SF and Ddimer levels were elevated at hospitalization and apixaban therapy with heparin could not reduce the SF level to normal. The patient developed Ischemic stroke under these conditions. After starting dabigatran therapy with heparin, his SF level returned to normal. For this patient, dabigatran therapy with heparin might have been more effective for inhibiting hypercoagulability than apixaban therapy with heparin. Elevated SF levels may indicate hypercoagulability during anticoagulation therapy; if a patient's SF level was elevated during anticoagulation therapy, it may indicate the necessity of selecting a different anticoagulant agent.

We thought that hypercoagulability of this patient was due to his lung cancer. When NVAF patients developed malignancy, it is necessary to maintain strict anticoagulation therapy against hypercoagulability to aid in preventing thrombosis $[17,18]$.

\section{Conclusions}

We report the case of a patient with NVAF-emergent PTE, DVT, and LAA thrombus during warfarin therapy, with subsequent ischemic stroke during apixaban therapy. The patient's SF level increased despite anticoagulation therapy. Monitoring SF values may be effective in evaluation of the hypercoagulable condition in anticoagulant therapy, and may serve as an index for changing anticoagulants.

\footnotetext{
Abbreviations

LAA: Left atrial appendage; NVAF: Non-valvular atrial fibrillation; PTE: Pulmonary thromboembolism; DVT: Deep vein thrombosis; NOACs: Non-vitamin K antantagonist oral anticoagulants; SF: Soluble fibrin; TTR: Time in therapeutic range; CT: Computed tomography; t-PA: Tissue plasminogen activator; INR: International normalized ratio; PT: Prothrombin time.
}

\section{Competing interests}

The authors declare that they no competing interests.

\section{Authors' contributions}

TK: Concept/design, Data analysis/interpretation. NT: Data collection. HI: Data collection. HO: Data collection. All authors read and approved the final manuscript.

\section{Author details}

'Division of Laboratory Medicine, Ichinomiyanishi Hospital, 1 Kaimei-hira, Ichinomiya, Aichi 494-0001, Japan. ²Division of Cardiology, Ichinomiyanishi Hospital, 1 Kaimei-hira, Ichinomiya, Aichi 494-0001, Japan.

Received: 2 December 2014 Accepted: 18 May 2015

Published online: 06 July 2015

\section{References}

1. JCS Joint Working Group. Guidelines for the diagnosis, treatment and prevention of pulmonary thromboembolism and deep vein thrombosis (JCS 2009). CirC J. 2011;75:1258-81.

2. Ogawa S, Aonuma K, Tse H-F, Huang D, Huang J-L, Kalman J, et al. The APHRS's 2013 statement of antithrombotic therapy of patients with nonvalvularatrial fibrillation. J Arrhythmia. 2013;29:190-200.

3. The European Atrial Fibrillation Trial Study Group. Optimal oral anticoagulant therapy in patients with nonrheumatic atrial fibrillation and recent cerebral ischemia. N Engl J Med. 1995;333:5-10.

4. Anderson JL, Halperin JL, Albert NM, Bozkurt B, Brindis RG, Curtis LH, et al. Management of patients with atrial fibrillation (compilation of 2006 ACCF/AHA/ESC and 2011 ACCF/AHA/HRS recommendations): a report of the American College of Cardiology/American Heart Association Task Force on Practice Guidelines. J Am Coll Cardiol. 2013;61:1935-44.

5. Connolly SJ, Ezekowitz MD, Yusuf S, Eikelboom J, Oldgren J, Parekh A, et al. Dabigatran versus warfarin in patients with atrial fibrillation. N Engl J Med. 2009;361:1139-51.

6. Granger CB, Alexander JH, McMurray JJ, Lopes RD, Hylek EM, Hanna M, et al. Apixaban versus warfarin in patients with atrial fibrillation. N Engl J Med. 2011;365:981-92.

7. Mitusch R, Siemens HJ, Garbe M, Wagner T, Sheikhzadeh A, Diederich KW. Detection of a hypercoagulable state in nonvalvular atrial fibrillation and the effect of anticoagulant therapy. Thromb Haemost. 1996;75:219-23.

8. Wells PS, Anderson DR, Rodger M, Forgie M, Kearon C, Dreyer J, et al. Evaluation of D-dimer in the diagnosis of suspected deep-vein thrombosis. N Engl J Med. 2003;349:1227-35.

9. Ruiz-Gimenez N, Friera A, Artieda P, Caballero P, Sanchez Molini P, Morales M, et al. Rapid D-dimer test combined a clinical model for deep vein thrombosis. Validation with ultrasonography and clinical follow-up in 383 patients. Thromb Haemost. 2004;91:1237-46.

10. Ota S, Wada H, Nobori T, Kobayashi T, Nishio M, Nishioka Y, et al. Diagnosis of deep vein thrombosis by plasma-soluble fibrin or D-dimer. Am J Hematol. 2005;79:274-80.

11. Palareti G, Cosmi B, Legnani C, Tosetto A, Brusi C, lorio A, et al. D-dimer testing to determine the duration of anticoagulation therapy. N Engl J Med. 2006;355:1780-9.

12. Wada H, Kobayashi T, Abe $Y$, Hatada T, Yamada N, Sudo A, et al. Elevated levels of soluble fibrin or D-dimer indicate high risk of thrombosis. J Thromb Haemost. 2006;4:1253-8.

13. Dempfle CE. The use of soluble fibrin in evaluating the acute and chronic hypercoagulable state. Thromb Haemost. 1999;82:673-83.

14. Tsuji A, Wada H, Matsumoto T, Abe Y, Ota S, Yamada N, et al. Elevated levels of soluble fibrin in patients with venous thromboembolism. Int J Hematol. 2008;88:448-53.

15. Sudo A, Wada H, Nobori T, Yamada N, Ito M, Niimi R, et al. Cut-off values of D-dimer and soluble fibrin for prediction of deep vein thrombosis after orthopaedic surgery. Int J Hematol. 2009;89:572-6.

16. Kawakami T, Kobayakawa H, Ohno H, Tanaka N, Ishihara H. Resolution of left atrial appendage thrombus with apixaban. Thromb J. 2013;11:26.

17. Hu YF, Liu CJ, Chang PM, Tsao HM, Lin YJ, Chang SL, et al. Incident thromboembolism and heart failure associated with new-onset atrial fibrillation in cancer patients. Int J Cardiol. 2013;165:355-7.

18. Farmakis D, Parissis J, Filippatos G. Insights into onco-cardiology: atrial fibrillation in cancer. J Am Coll Cardiol. 2014;63:945-53. 